Haoyun Tang

Huilong Ren

Qi Zhong

http://dx.doi.org/10.21278/brod70206

ISSN 0007-215X

eISSN 1845-5859

\title{
DESIGN AND MODEL TEST OF STRUCTURAL MONITORING AND ASSESSMENT SYSTEM FOR TRIMARAN
}

\author{
UDC 629.5.022.3:629.5.015.4:629.5.018.4
}

Original scientific paper

\begin{abstract}
Summary
Due to the unique structural design of multihull, the structural response on trimaran is often more complex than the response of monohull in navigation. In order to ensure the safety of trimaran navigation and predict the potential damage of local structure, the structure monitoring technology is applied. According to the structural characteristics of trimaran, structural monitoring and assessment system for multihull is designed and introduced in detail, and corresponding model test is taken to demonstrate its effectiveness. The selfpropelled trimaran model is installed with sensors in different longitudinal positions to monitor the variety of structural responses in irregular waves. And three real-time structural strength assessment methods in the system are used respectively to indicate the structural state about hull longitudinal strength, local yielding strength and fatigue strength. The influence of different wave azimuths and monitoring positions on structural strength assessment is analysed. Finally, these measured data and analyses results will provide technical support for design and installation of the monitoring system on actual trimaran.
\end{abstract}

Key words: $\quad$ Trimaran; Structure monitoring; structure strength; model experiment; irregular wave; fatigue damage

\section{Introduction}

With the development of structure monitoring technology, the hull safety monitoring has been paid more and more attention in shipbuilding industry. The real-time strain on hull structure is observed and analysed by the arrangement of sensors in some critical locations. And the corresponding assessment based on measured data is taken to guide the crews to avoid the structural failure accidents in complex sea environment. The application of hull safety monitoring system into ship has been regard as an effective mean to improve the survivability of ship navigation in harsh sea environment.

In fact, many scholars and research institutions had carried out a series of experiments and correlative researches on hull monitoring technology. Lindemann firstly applied the structure monitoring technology into ships in the late 70's in twentieth Century [1]. Although his research had not achieved the desired results with the limit of computer and sensor 
technology, it gave a concise way to build hull monitoring system. In twenty-first Century, the computer technology and related electronic data transmission research had made great progress. And the technology of structure monitoring on ships was more feasible. In 1994, the Bureau veritas and Kelvin Hughes Company jointly developed a kind of hull monitoring system under the European project of Brite-EuRam II [2]. The system could monitor the ship structural stress in its whole life, but the algorithm towards structural response in the system is incomplete. It lacks the extraction and processing of high-frequency hull vibration. Then, Hjelme (1997) tried to use two kinds of fibre-optical sensors in model test to catch the structural response of hydroelasticity and bow slamming [3]. In his research, the advantages of fiber-optical Bragg grating strain sensor in the field of hull structure monitoring were illustrated, and the characteristics of the high-frequency structural response were observed. After the Hjelme's research about application of fibre-optical sensors in structural monitoring, the American Naval Research Laboratory (NRL) combined with Norwegian Defense Research Establishment (NDRE) to study systematically the structural stress monitoring system with fibre optic sensors from 1997 to 2001. The developed monitoring system was applied respectively in ship experiment of Skjold-class fast patrol boat and Oksøy/Alta-class mine counter measure vessels [4-6]. In the ship tests, the bending moment and wave slamming was monitored by the fibre optic sensors installed at the bow and midship section. In 2003, an American high-speed catamaran called "HSV-2 Swift" was also installed a new-style structural stress monitoring system by the united research group, which included the United States Naval Operations Center, Georgia Institute of Technology, Michigan University and Lehigh University. This real-time monitoring system focused on the wave force and vibration on the aluminium alloy hull structure, and acceleration was firstly included in the real-time monitoring system as an important monitoring parameter [7-8]. Although the ship test had achieved good monitoring results in this stage, there was still a lack of structural evaluation methods to reflect the structural safety status of ships. Base on the theoretical and experimental research on the hull structural monitoring system using fibre optic sensors, Nielsen studied the structural fatigue strength computing method in a container monitoring system. At the same time, the ship motion and environment data such as vertical acceleration, sailing speed, wave height, wind speed and direction, was also recorded in the system [9]. These data laid the foundation on the improvement of container ship design. And it also demonstrates the value of the monitoring system in civilian ships. In order to ensure safe navigation of civilian ships further, Renato developed a simplified assessment method to monitor the longitudinal strength of hull girder using the stress monitoring system [10]. This processing method of monitoring data could be used as a reference for ship emergency. Then, Kokarakis studied a kind of structural corrosion predict method in the monitoring system for bulk carriers. This system could correct and predict the structural corrosion by monitoring the strain [11]. Data communication technology is also very important in the structural monitoring system. It directly affects the efficiency and stability of the monitoring system. Based on CAN communication technology, Yang designed a kind of data transmission framework to make the structural stress monitoring system more convenient and efficient [12]. In addition, He developed a method by combining the multi-channel cross sampling technology and optical fiber sensing technology to monitor the ship deck deformation in realtime. Its feasibility was proved by the experiment and the simulation calculation [13]. Based on research in data communication technology and structural monitoring technology, Jin developed a kind of Container Ship Structural Monitoring and Evaluation System (CSSMAS) and putted it into actual ship experiment [14]. The container ship called "YuFeng" installed some strain sensors and acceleration sensors to monitor local structure condition. Meanwhile, the encountering waves around this container ship were also monitored by the image sensors 
at the fore and aft of the container ship. The results of ship test were satisfactory, and the data was also used to optimize container ship design.

Although the hull structural monitoring technology has been applied widely, there are still many technical difficulties in the field of hull structure monitoring. In fact, most structural monitoring systems are designed by simple and direct requirement scheme. It has a high requirement for computer space, and it is not suitable for long-term and high-speed hull structure monitoring. In order to ensure the monitoring system stability, the number of monitoring sensors in the system is often limited to less than 10 , so that the overall longitudinal structural strength monitoring and local structural strength monitoring towards ships cannot be fully considered at the same time. Due to the limitations of the monitoring sensor number, most systems mainly monitor the hull structure at bow and midship, recording and analysing the bow slamming and hull longitudinal structural strength. For some local structural monitoring systems, the monitoring positions are always selected in a small range, and the longitudinal structural strength assessment is often abandoned. So these systems are not easy to expand its functions. Obviously, a multiple composite structure is needed in the design of monitoring system. Parallel computing technology and efficient data processing method should be applied to improve monitoring system stability. In the research on the realtime structural strength evaluation, the existing systems often adopt a simple deterministic evaluation method. Although this deterministic calculation method is fast, the possibility of the sudden wave load changing is neglected. Therefore, a series of structural reliability assessment methods based on real-time monitoring data is needed to ensure the structural assessment by different structural destruction forms. In addition, the most structural monitoring researches mainly focused on traditional monohull and catamaran. There is a lack of the structural monitoring research towards trimaran. As a new-style multihull, trimaran not only has excellent seakeeping performance, but also suffers complex wave load response in irregular waves. The high sailing speed and application of new materials on trimaran increase the structural failure risk in further. Therefore, there is an urgent need to develop a structural monitoring and assessment system for trimaran.

In this paper, Structural Monitoring and Assessment System (SMAS) for trimaran is designed and developed. The multi-level and modular design method is used to make the system run flexibly and expand easily. In order to speed up the system operation further, multi-thread parallel technology is also applied. And the real-time structural strength assessment method, such as simplified yield strength assessment, longitudinal strength assessment, fatigue strength evaluation, is studied to meet the need for overall longitudinal structural strength monitoring and local structural strength monitoring. By constructing the corresponding probability distribution of wave load and structure resistant ability, the structural strength reliability method is studied. In order to improve the utilization of system space, the monitoring system adopts the data processing technology of partitioned storage and cyclic coverage. By combining a variety of technologies, the trimaran structural monitoring system can monitor the hull longitudinal strength and local structural strength simultaneously, and the number of connected monitoring sensors can reach up to 60. In addition, in order to demonstrate the feasibility and effectiveness of this system, the trimaran model equipped with SMAS is tested with different wave azimuths. The real-time fluctuation of strain and sectional monitoring values is recoded in monitoring system. And structural strength assessments are worked in the system to evaluate the structural state in short term and long term. Through comparison and analysis of the assessment results in different wave azimuths, the dangerous positions and its structural damage cause are found. Finally, the measured data and assessment result will become an important foundation on trimaran detailed optimization design. 


\section{Design of structural monitoring and assessment system}

\subsection{Overall design of structural monitoring and assessment system}

The overall design of structural monitoring and assessment system is a key part in the system design. It can not only affect the system computing efficiency, but also determines the scalability of its functions. In order to make the monitoring system run and extend more efficiently, the multi-level and modular design method is applied in the overall design of trimaran monitoring system, as seen in Figure 1. Base on the structural monitoring purposes, the whole monitoring system is divided into three modules, which are real-time structural monitoring module, structural strength assessment module and structural monitoring database module. Each module owns its sub-modules to implement their specific functions.
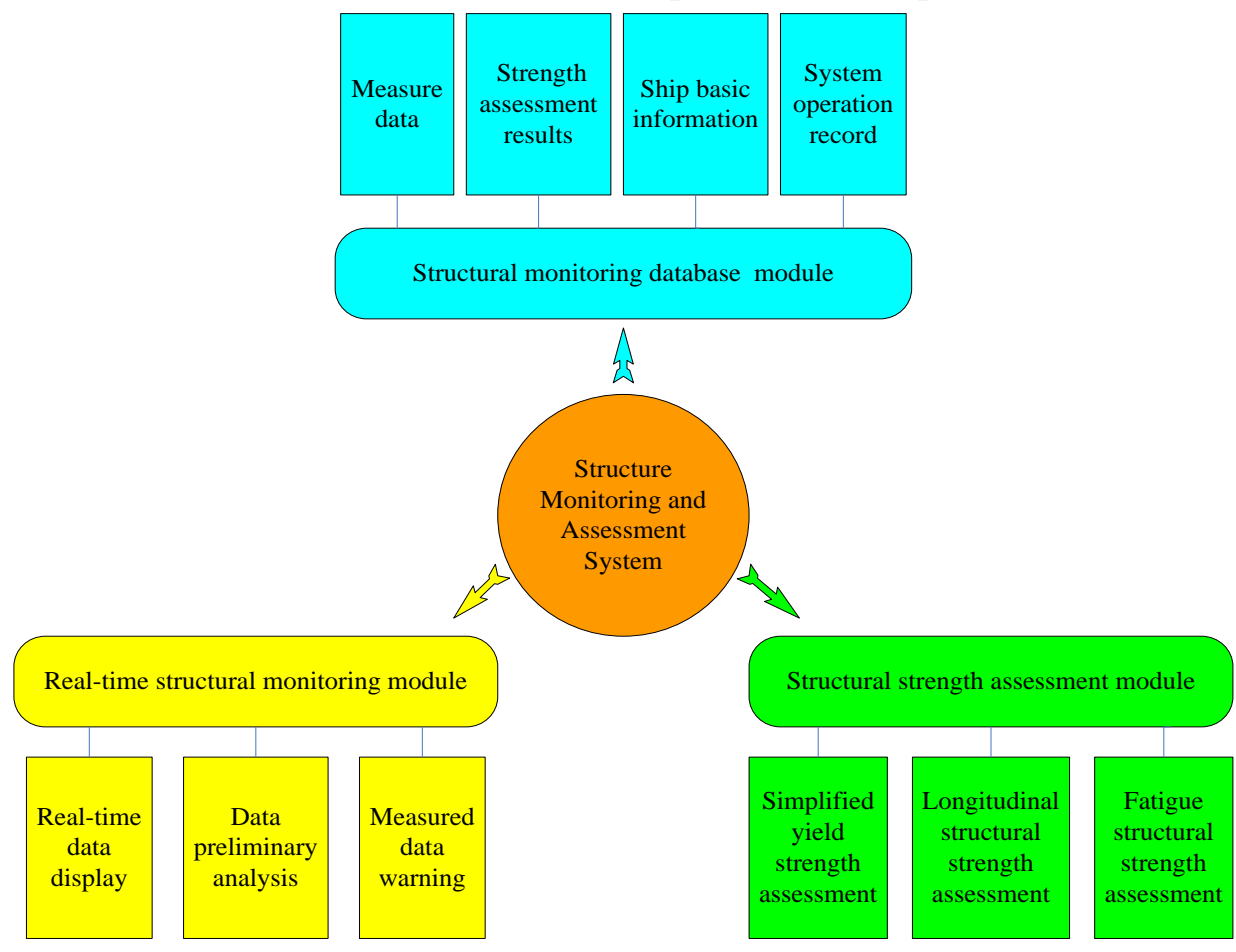

Fig. 1 Scheme of monitoring system

In real-time structural monitoring module, the real-time data of structure response is focused on. The fluctuation of structural response like strain can be recorded and showed by curve graph and histogram. The preliminary analysis and measured data warning is also taken in this module. In preliminary analysis, the statistical characteristic value such as significant amplitude values, maximum and mean value is calculated and guided to help crew understand the basic parameters of structure response more clearly. When the structural responses are violent and beyond to designed alarms threshold, the warning message will appear and point out the warning location. And the out-of-range proportion on the warning threshold is record and store into structural monitoring database. In structural strength assessment module, all programs are worked around calculation and display about the structural strength state. The structural strength assessments in this module include the longitudinal structural strength assessment, fatigue structural strength assessment and simplified yield strength assessment. The characteristics of trimaran structural strength state in different time-domain are recoded by these methods. The short-term structure state is evaluated by longitudinal structural strength assessment. And the instantaneous dangerous structure state is recoded by simplified yield strength assessment. The potential damage of structural strength state in long term is also monitored by the fatigue structural strength assessment. Structural monitoring database is 
an assemblage of measured structural response and strength assessment results. The ship basic information and system operation record is also added in this database. Finally, this database forms a complete logbook about trimaran's structural state.

Through the actual ship test and preliminary research, it is known that the simple singlethreaded calculation method often takes too much time and limits the number of monitoring sensors. In order to make the trimaran monitoring system work more efficiency, multithreading parallel computing method is adopted. Figure 2 shows the multi-threading scheme of the monitoring system. Five threads are designed for the trimaran monitoring system. They are data collection and display thread, data extraction and storage thread, longitudinal structure strength thread, simplified yield strength thread and fatigue structure strength thread. Each thread extracts and analyses the related data independently, then finishes its own function.

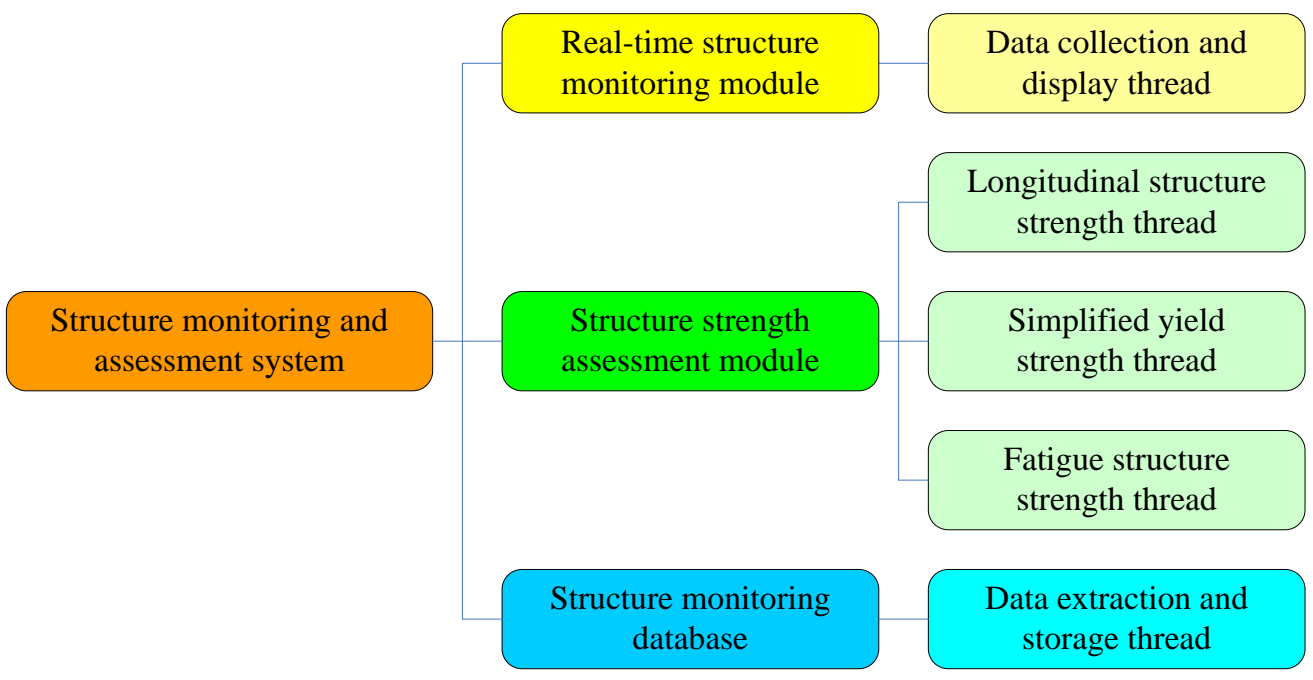

Fig. 2 Multi-threading scheme of monitoring system

The real-time structural monitoring module is mainly supported by the data collection and display thread. Firstly, this thread receives and classifies the measured data from the strain sensors by using the Transmission Control Protocol (TCP)/IP services. Through combining with the correlation coefficient, the electrical signals are converted into the monitoring variables such as strain, bending moment and torque. And these real-time monitoring variables are shown in histogram or diagram to express the fluctuation of structural response directly. The maximum and mean value is also calculated at same times. Then, the judgement of data warning is taken. If the monitoring indicators appear large values, the data collection and display thread will feed back to data extraction and storage thread. Finally, the data extraction and storage thread will extract corresponding location information and give warning toward crew. In structural strength assessment module, the three kinds of structural strength assessment use their own thread. There is no interference between these threads, so it improves the calculation speed of assessment system. The longitudinal strength thread is based on the strength reliability method to assess hull sectional structure state at different longitudinal positions. The strength thread counts time and calculates the structural failure probability every 30 minutes. The simplified yield strength thread is aimed to evaluate instantaneous structure state. The max strain value in preliminary analysis is transmitted by data collection and display thread to simplified yield strength thread. And the instantaneous yield indictor is calculated and classified into corresponding grades. The fatigue structural strength thread is based on the Palmgren-Miner damage rule, and run itself every 5 minutes. The potential damage of structural fatigue failure is measured by the fatigue cumulative damage. The fatigue damage in $5 \mathrm{~min}$ is calculated and added with previous fatigue damage 
to become final fatigue cumulative damage in fatigue structural strength thread. In addition, all assessment results will be written into database by their own threads. In structural monitoring database, the duty of data extraction and storage thread is to write and update the data in structural monitoring database. All query and extraction of data from monitoring database is worked by this thread. The extraction and storage thread also record the crew system operation and abnormal monitoring data for subsequent accident analysis. Through using these threads, the monitoring system can reflect the structure state and deal with some emergency incident quickly. In fact, the max sampling frequency of SMAS can reach $1000 \mathrm{~Hz}$. And the fluctuation of sensor sampling frequency, which is from $10 \mathrm{~Hz}$ to $1000 \mathrm{~Hz}$, is also supported automatically by this trimaran monitoring system. Figure 3 shows the designed results of the structural monitoring and assessment system.

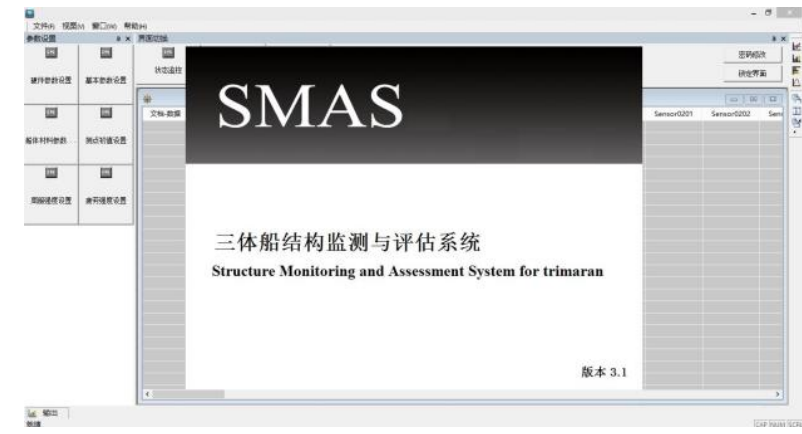

(1) Operation of SMAS

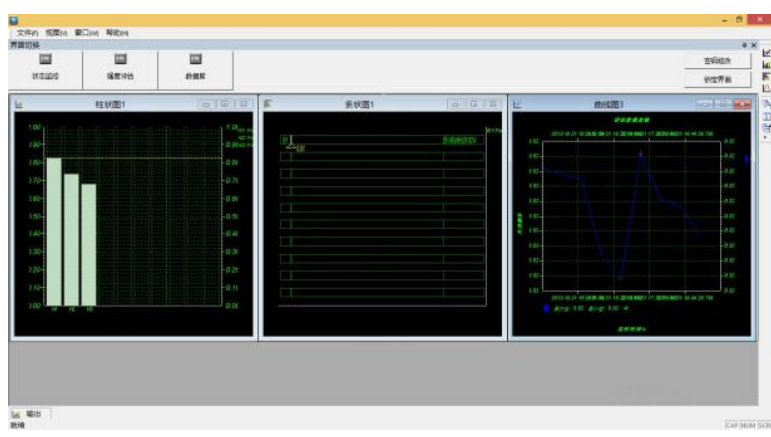

(3) Structure strength assessment module

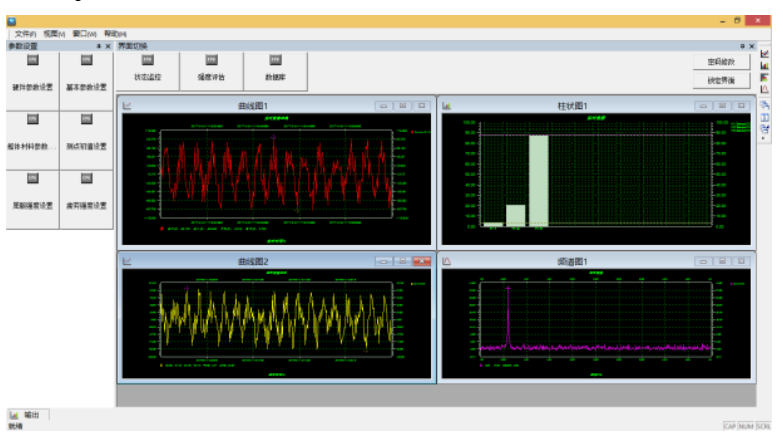

(2) Real-time structural monitoring module

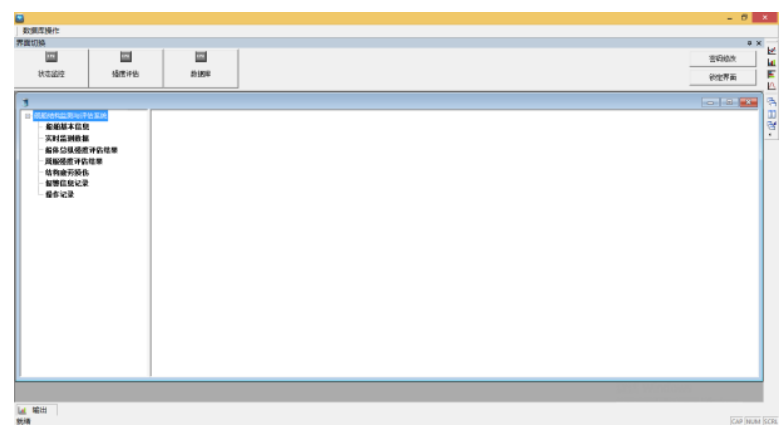

(4) Structural monitoring database module

Fig. 3 Structural monitoring and Assessment System for tirmaran

\subsection{Structural strength assessment principle in monitoring system}

Structural strength assessment based on real-time data has always been a difficult point in the field of structural monitoring. Based on the research of structural real-time monitoring technology, this paper gives some real-time structural strength assessment methods for trimaran structure. These methods include the deterministic assessment method and reliability assessment method. And, considering characteristics of different structural damage form, the system adopts three methods to achieve different structural strength monitoring targets.

\subsubsection{Simplified yield strength assessment}

In yield strength assessment, a simplified deterministic evaluation method is studied in the paper. This method requirement of data quantity is relatively small, and the computing speed is fast. Therefore, this assessment method is beneficial to reflect instantaneous structure condition. Some critical structures, which suffer the structural response frequently and violently, are suitable to use this assessment method. In simplified yield strength assessment, the instantaneous yield indictor $S(t)$ records the drastic structural state fluctuation and 
occurrence time. This indictor is calculated as a ratio between the max value of real-time measured stress $\sigma_{m}(t)$ and the allowable stress of material $\left[\sigma_{s}\right]$, as shown in Eq. (1).

$$
S(t)=\frac{\sigma_{m}(t)}{\left[\sigma_{s}\right]}
$$

Due to differences in material production, the yield limit of the material is not always invariant constant. It is generally considered that the yield limit of the material fits Gaussian distribution. According to the statistical theory on Gaussian distribution, the probability of yield limit in the area of $(\mu-2 \sigma, \mu+2 \sigma)$ is $95.44 \%$. Obviously, the possibility of yielding failure on structure will increase greatly, when the real-time measured stress come into this area. Therefore, the allowable stress of material $\left[\sigma_{s}\right]$ is often below to yield limit of material, and is defined as follows.

$$
\left[\sigma_{s}\right]=\mu-2 \sigma
$$

where $\sigma$ is standard deviation on material yield limit. And $\mu$ is mean value on material yield limit. Then, the structural safety classification based final instantaneous yield indictor is taken. The specific ranges of different grades are shown in Table 1.

Table 1 Sructure safety classification

\begin{tabular}{|c|c|}
\hline Safety grade & Range of instantaneous yield indictor \\
\hline Class A & $0 \% \sim 1 \%$ \\
\hline Class B & $1 \% \sim 10 \%$ \\
\hline Class C & $10 \% \sim 100 \%$ \\
\hline Class D & $>100 \%$ \\
\hline
\end{tabular}

\subsubsection{Longitudinal strength assessment}

For longitudinal strength assessment, a kind of structural strength reliability method is studied in this paper to evaluate the sectional structure condition. In order to observe the monitoring structural response in real time, the sensors are often installed on hull girder or some longitudinal stiffened panels at different longitudinal positions. The relationship between monitoring structure response and sensor signals is built by some calibration experiments [15]. And the structural section response like bending moment and torque can be monitored. In structural reliability method, the structural response caused by wave impact is representing as $D$. And $C$ is function of the structural resistant ability. When $C$ and $D$ are independent random variables, the structural failure function $M$ can be defined as follows.

$$
M=C-D
$$

Obviously, the structure breaks down, when the structure failure function $M$ is less than 0 . Therefore, structural failure probability $P_{f}$ is calculated out by Eq. (4).

$$
P_{f}=P(M<0)=\int_{-\infty}^{+\infty}\left[1-F_{D}(x)\right] f_{C}(x) d x
$$

Where $x$ is the value of structural response, $f_{C}(x)$ is the probability density function on the structural resistant ability, and $F_{D}(x)$ is the distribution function on extreme value of structural response. According to the statistical data of wave load, the short term distribution on the amplitude of structural response often follows the Rayleigh distribution. So the extreme value distribution of structural response is achieved on the foundation of Rayleigh distribution. The Rayleigh distribution for structural response in short term is shown in Eq. (5). 
And the computational method on the corresponding parameter of Rayleigh distribution $k$ is also given in Eq. (6).

$$
\begin{gathered}
F_{d}=1-\exp \left(-\frac{x^{2}}{k^{2}}\right) \\
k^{2}=2 \sigma_{d}^{2}
\end{gathered}
$$

Where $\sigma_{d}$ is the standard deviation of real-time structure response, and it is statistically calculated every 30 minutes. The cyclic number of real-time data $n$ is also counted. According to the principle of sequence statistics, the distribution of the extreme value on structural response can be obtained as follows.

$$
F_{D}=\left[1-\exp \left(-\frac{x^{2}}{k^{2}}\right)\right]^{n}
$$

The ability of structural resistance is considered generally to obey Gaussian distribution. Therefore, the probability density function on the structural resistant ability $f_{C}(x)$ is calculated by Eq. (8).

$$
\begin{array}{r}
f_{c}=\frac{1}{\sqrt{2 \pi} \sigma_{c}} \exp \left(-\frac{\left(x-\mu_{c}\right)^{2}}{2 \sigma_{c}}\right) \\
\mu_{c}=\mu_{s}-d_{i}
\end{array}
$$

Where $\sigma_{c}$ is the standard deviation of the probability density function on the structural resistant ability; $\mu_{c}$ is mean value of the probability density function; $d_{i}$ is the fluctuation of monitoring data caused by other factors such as sensor temperature, changing of ship loading status and the initial stress in materiel. The mean value $\mu_{c}$ can be obtain by subtracting $d_{i}$ from section ultimate strength $\mu_{s}$ as Eq. (9).

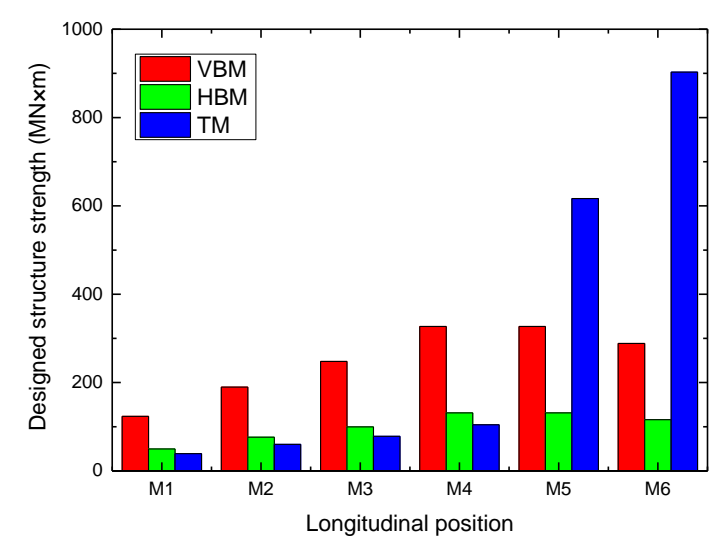

Fig. 4 Results of designed structure strength

In fact, the trimaran ultimate strength at different cross-sections needs to consider many factors such as structural failure forms and material yield characteristics, so the accuracy of ultimate strength calculation is difficult to guarantee. And the Rule for the Classification of Trimaran is issued by the Lloyd's Register (LR) in 2006, it is unique complete rule for trimaran design [16]. Most trimarans are designed and built on the basis of this rule. In LR's trimaran rule, the calculation formula of hull structure strength is given. And the requirements of hull structural strength in each cross-section must be satisfied. Therefore, the ultimate 
strength at cross-section in this paper is simplified and calculated by LR's rule. Figure 4 show the designed structural ultimate strength in different locations. Then, the mean value of structural resistance can be obtained by eliminating non-wave disturbance. And the coefficient of variation on structural resistance distribution is assumed as $8 \%$, according to previous research on hull ultimate strength [17]. The structural resistance ability function at different cross-sections is established. Finally, the longitudinal strength assessment in monitoring system works successfully. Figure 5 shows the process of longitudinal strength assessment in monitoring system.

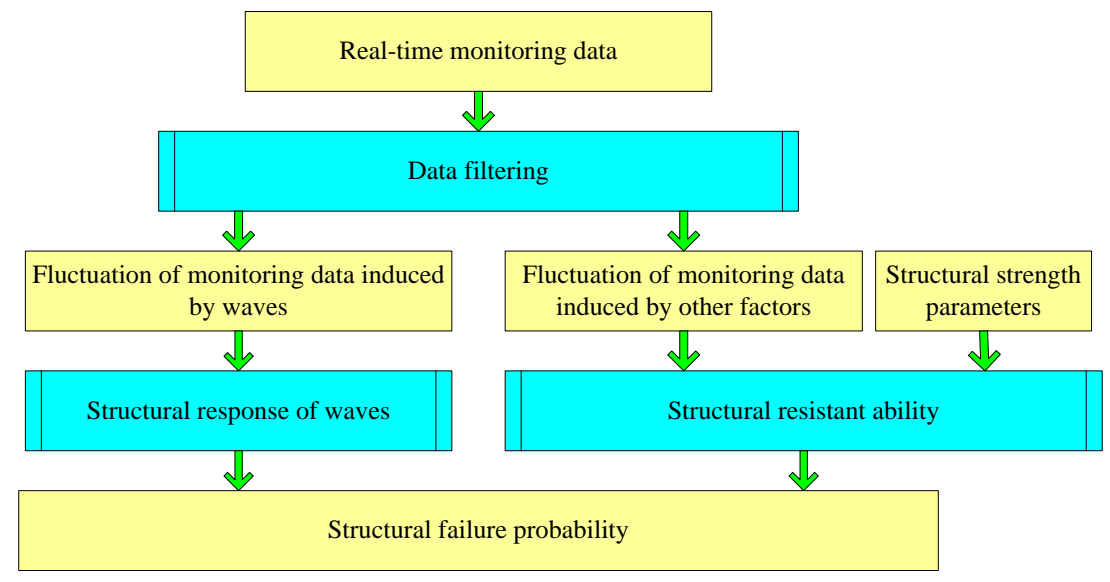

Fig. 5 Process of longitudinal strength evaluation

\subsubsection{Fatigue strength assessment}

The structural fatigue failure is easy to occur, when the ship suffers the high-frequency wave load. It is also a kind of potential structural failure form that is often overlooked. Nowadays many ship accidents are found to be caused by structural fatigue. Therefore, the structural fatigue monitoring is indispensable. Based on the Palmgren-Miner damage rule and the rain flow counting method, a kind of real-time fatigue strength assessment is studied.

In fact, the fluctuation of stress is always irregular and random, when ship sails in the complex irregular wave condition. Therefore, the rain flow counting method is needed to transform the random stress into a series of variable amplitude stresses. And the characteristic parameter of these variable amplitude stresses, which includes the cyclic number, amplitude and mean value, is also calculated out by the rain flow counting method. Because the S-N curve method is based on symmetrical stress cycle condition, these measured asymmetric stresses need to be corrected in further. So the Gerber correction method, which is applied widely in ship engineering, is used. The equation of the Gerber correction is as follows.

$$
\frac{S_{a}}{S_{r}}+\frac{S_{m}^{2}}{S_{y}^{2}}=1
$$

Where $S_{a}$ is the amplitude of measured stress; $S_{r}$ is the corrected amplitude of stress under symmetrical cycle; $S_{m}$ is mean value of measured stress; And $S_{y}$ is ultimate strength stress, it is depended on material yield limit. The corrected amplitude of stress under symmetrical cycle $S_{r}$ is calculated by solving the Eq.(10). Then, the maximum cyclic number of structural fatigue limit under the corrected amplitude stress $N$ can be calculated by the S-N curve method as follows.

$$
\log (N)=\log (K)-m \log \left(S_{r}\right)
$$


Where $K$ is the $S$ - $N$ curve parameter. And $m$ is the inverse slope of the $S-N$ curve. These parameters of $S-N$ curve are determined by the material fatigue test [18] and guide for fatigue strength of hull structures in China Classification Society [19]. Then, the ratio between the measured cyclic number $n_{i}$ and the cyclic number of the corresponding fatigue limit $N_{i}$ is calculated on each series of variable amplitude stresses. And the final fatigue cumulative damage $D_{f}$ is achieved by summing these ratios, as shown as Eq. (12).

$$
D_{f}=\sum_{i=1}^{j} \frac{n_{i}}{N_{i}}
$$

Thereinto, $j$ is the number of the series of variable amplitude stresses; $n_{i}$ is the measured cyclic number on the $i$ th series of variable amplitude stresses, it is obtained by rain flow counting method; $N_{i}$ is the cyclic number of the corresponding fatigue limit on the $i$ th stress level. The whole calculation process of fatigue strength assessment is shown at Fig. 6.

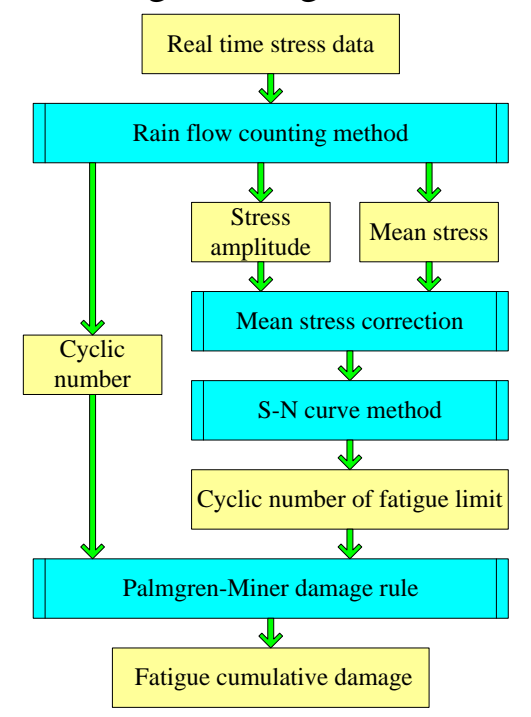

Fig. 6 Process of fatigue strength assessment

\subsection{Establishment of structural monitoring database}

To store monitoring data more conveniently, based on the Structured Query Language (SQL) server, a kind of data extraction and storage method is developed to establish a trimaran structural monitoring database. In order to record the trimaran structural information as much detail as possible, seven tables are designed in this monitoring database, as seen in Figure 7. Each table consists of time data and corresponding monitoring data.

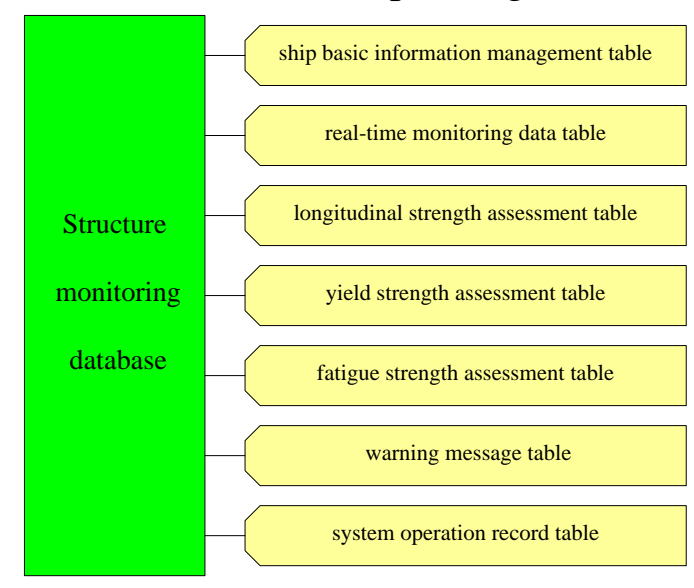

Fig. 7 Structure diagram of monitoring database 
The data extraction and storage thread plays a critical role in the data extraction and storage method for trimaran structural monitoring. It will be able to write the data into the corresponding table, and also extract the data from these tables when the monitoring data is inquired. In long-term structural monitoring, the huge quantity of monitoring data like realtime measured data is high possible to lower the system querying speed. Therefore, in order to avoid this influence, the partition function is used in each table. The storage space of table is divided into 48 regions. All region addresses are recorded by the partition function, so the system can find the corresponding storage location quickly. When all region storage is full, the thread will create some specified documents to output these data one region by one region. At same time, the method of cyclic covering region is also done to keep the number of regions unchanged. After the data output, the early stage region is regard as a new empty region to restart storage for the latest monitoring data. Certainly, the partition function still mark the outputted data addresses and can help the thread to find corresponding data in stored documents. Through system running test in long-term (six months), it is verified that the design of data storage improves the efficiency on data querying, and make the system speed of data extraction less than $15 \mathrm{~s}$ averagely. Figure 8 gives some results of the structural monitoring database design in SMAS.

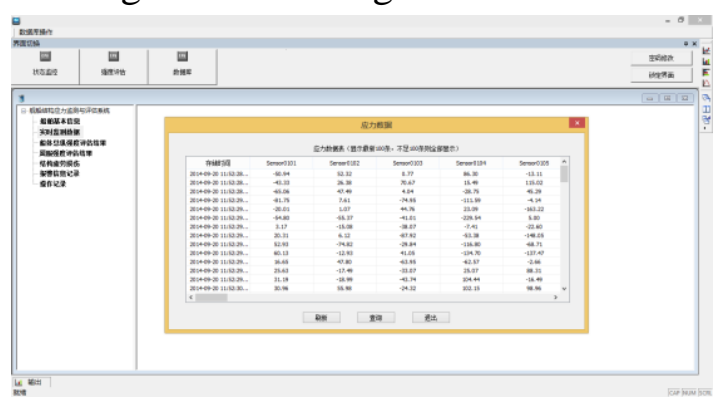

(1) Real-time monitoring data record

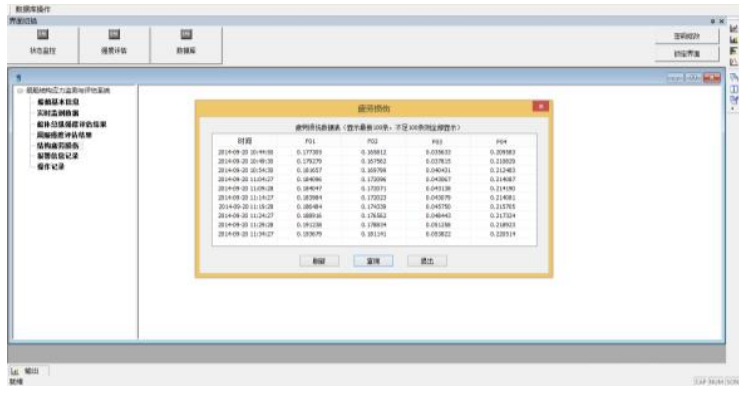

(2) fatigue strength assessment record

Fig. 8 Design of structural monitoring database

\section{Design of trimaran structural monitoring model}

In order to observe the characteristics of structural response in irregular waves and demonstrate the feasibility on structural monitoring system, a structural monitoring model is designed with the scale ratio of 1 to 25 by the model similarity theory [20-22]. The trimaran model had a series of segmented shells, which would offer similar wave force and impact to simulate the wave influence toward full-scale trimaran. And the disturbance of surround flow field caused by propeller is also considered by the installation of self-propulsion equipment in this trimaran model.

Table 2 Principal dimension of the trimaran model

\begin{tabular}{|c|c|}
\hline Description & Model \\
\hline Overall length $(\mathrm{m})$ & 5.64 \\
\hline Waterline length $(\mathrm{m})$ & 5.44 \\
\hline Moulded breadth $(\mathrm{m})$ & 1.06 \\
\hline Depth $(\mathrm{m})$ & 0.47 \\
\hline Draft $(\mathrm{m})$ & 0.2 \\
\hline Displacement $(\mathrm{t})$ & 0.255 \\
\hline
\end{tabular}


In addition, there are two kinds of steel backbones systems in the model. The longitudinal steel backbone system includes ten steel girders with different thickness to meet the stiffness distribution along the ship length. And transverse steel backbone system has two girders to ensure the transverse stiffness of cross-bridge structure. The proper placement of iron is taken to satisfy the similarity on mass distribution between the model and full-scale ship. Finally, the principal dimension of the trimaran model is designed and shown in table 2. And Figure 9 also shows the detailed design of trimaran model.

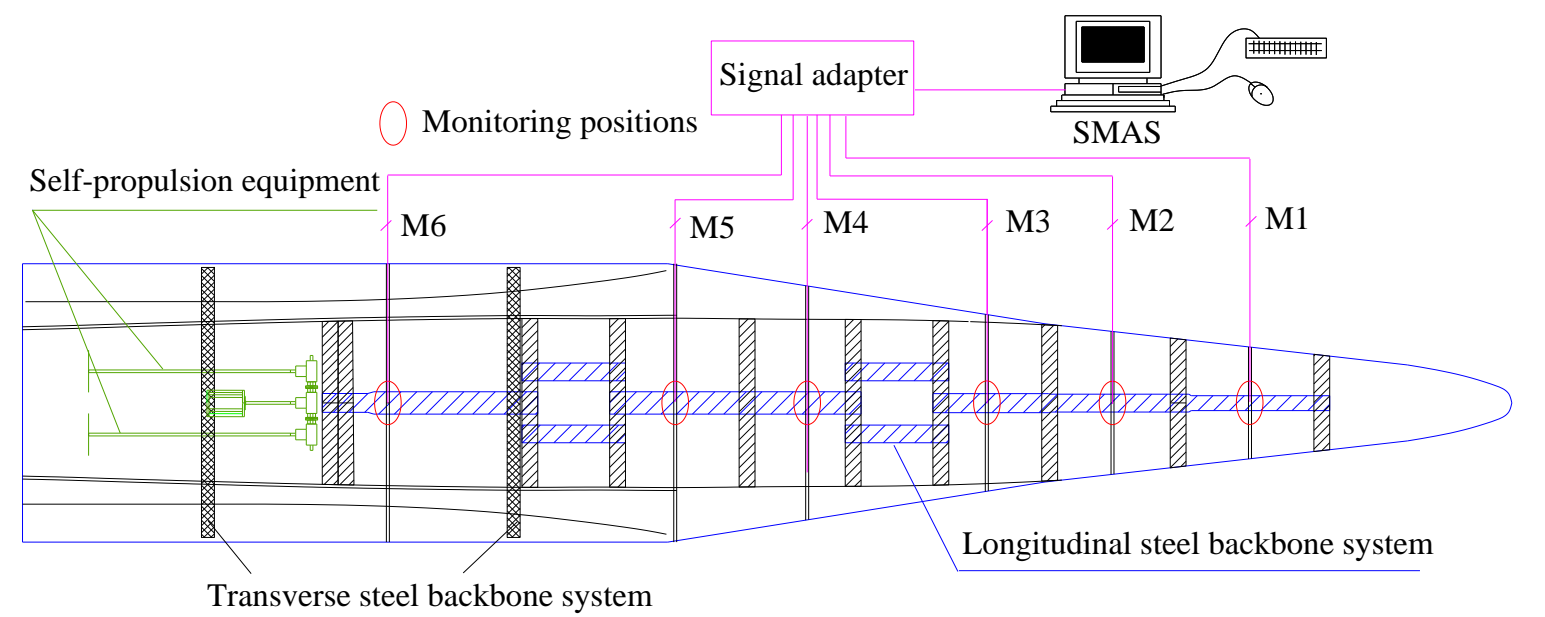

Fig. 9 Design of trimaran model

In Figure 9, it is observed that the model is divided into seven segments along the ship length. In order to measure the wave-induced hydroelastic vibration on the trimaran model, the interval in each segment maintain at $1 \mathrm{~cm}$. And the thin latex is used to keep the watertight in each interval. The positions of these intervals are introduced at table 3. And FP denotes fore perpendicular.

Table 3 Location of division

\begin{tabular}{|c|c|}
\hline Division & Location from FP $(\mathrm{m})$ \\
\hline M1 & 1.02 \\
\hline M2 & 1.56 \\
\hline M3 & 2.04 \\
\hline M4 & 2.72 \\
\hline M5 & 3.19 \\
\hline M6 & 4.28 \\
\hline
\end{tabular}

In fact, these interval positions are also the main structural monitoring points in the model test. The strain sensors are installed on the longitudinal circular hollow girders at each interval to monitoring the structural strain in model test. And the method of Wheatstone bridge was applied on the arrangement of the strain gauges to measure vertical bending moment (VBM), horizontal bending moment (HBM) and torque. The specific monitoring sensor layout and corresponding circuit diagram on the three structural deformations are shown respectively in Figure 10(a)-(c). In order to avoid the possible wire drag and water injection in model test, the silicon rubber is used around these monitoring sensors. And the installation process of these strain sensors is also seen in Figure 10(d). At same time, the relationship between the monitoring value and the output voltage of strain sensors is obtained by the static calibration experiment. Figure 11 shows the process and the results of static calibration experiment at bow. 


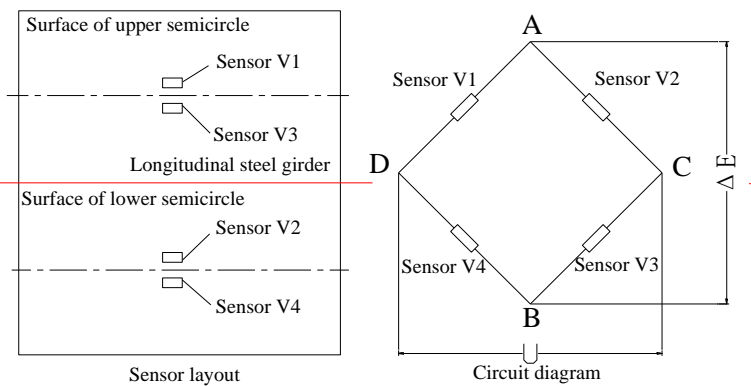

(a) Sensor arrangement for VBM

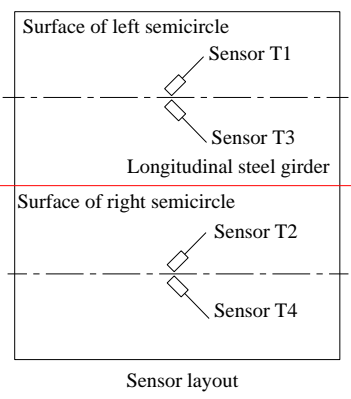

(c) Sensor arrangement for torque
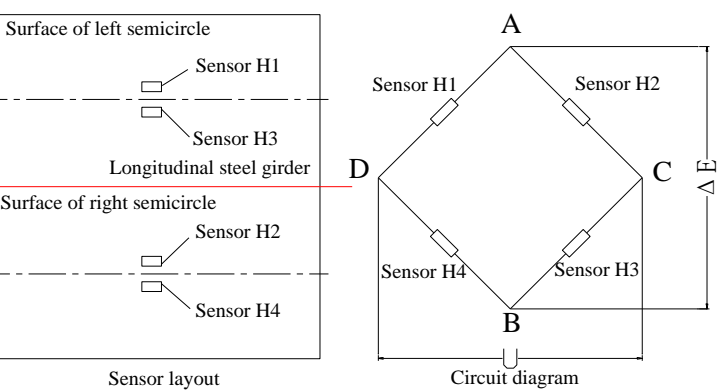

(b) Sensor arrangement for HBM

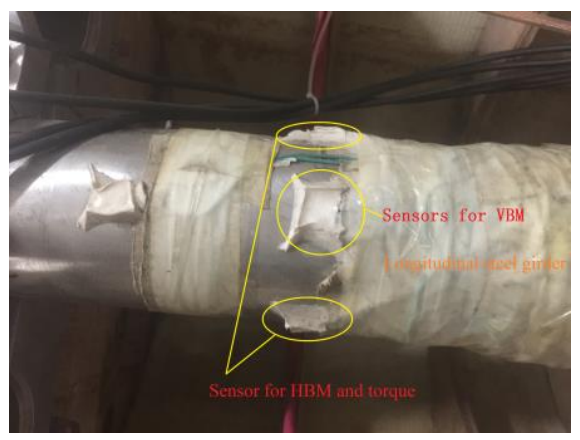

(d) Arrangement of sensors

Fig. 10 Arrangement and installation of strain gauges

In Figure 11 (a), it can be seen clearly that the measured steel girder in trimaran model was fixed with orthogonal metal frame at one end. At another end of steel girder, some weights were putted to form bending deformation or torsional deformation on the measured girder. The theoretical bending moment and torque at the monitoring position with different weights is calculated, and the output voltage of the monitoring sensors is also observed. By using linear fitting method, the corresponding correlation coefficients on the relationship between the sensor's output voltage and monitoring values like bending moment or torque are found out.

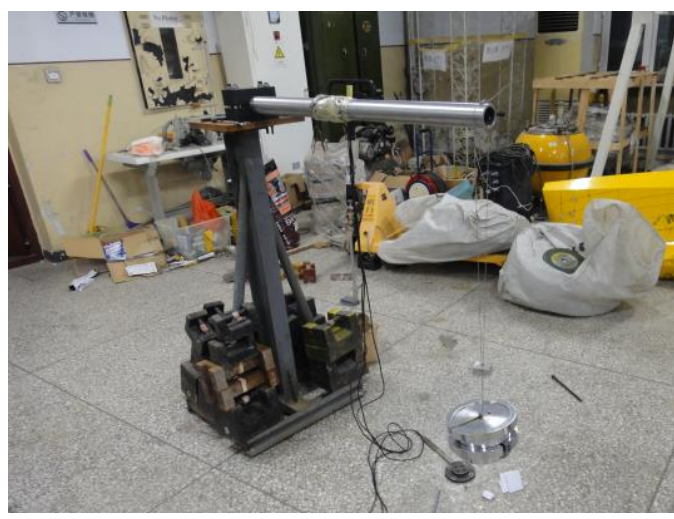

(a) Process of calibration experiment

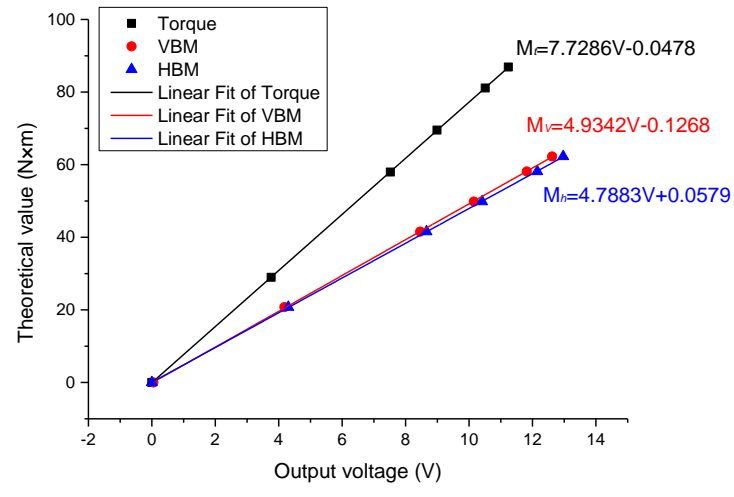

(b) Results of calibration experiment at M1

Fig. 11 Static calibration experiment

Figure 11 (b) shows the result of static calibration experiment at M1. The similar method is used to obtain corresponding correlation coefficient at other monitoring positions. And the correlation coefficient on surface strains also follows the same process in static calibration experiment. 


\section{Model test and monitoring results analysis}

The model test was taken in the comprehensive tank of Harbin Engineering University. And the ISSC dual parameter spectrum was used to guide the hydraulically driven wave maker for simulation of irregular waves. In model test, the typical irregular wave environment for full-scale trimaran, whose statistical significant wave height was $4 \mathrm{~m}$ and zero-crossing period was $6.7 \mathrm{~s}$, was mainly considered. By changing the model orientation, the measurement of model structural response in different wave azimuths was achieved. In addition, the model self-propulsion equipment was controlled to make the relative position invariant with trailer. Each irregular wave case lasted 2 hours to give enough time for structural strength assessment in SMAS. Figure 12 shows the process of model test.

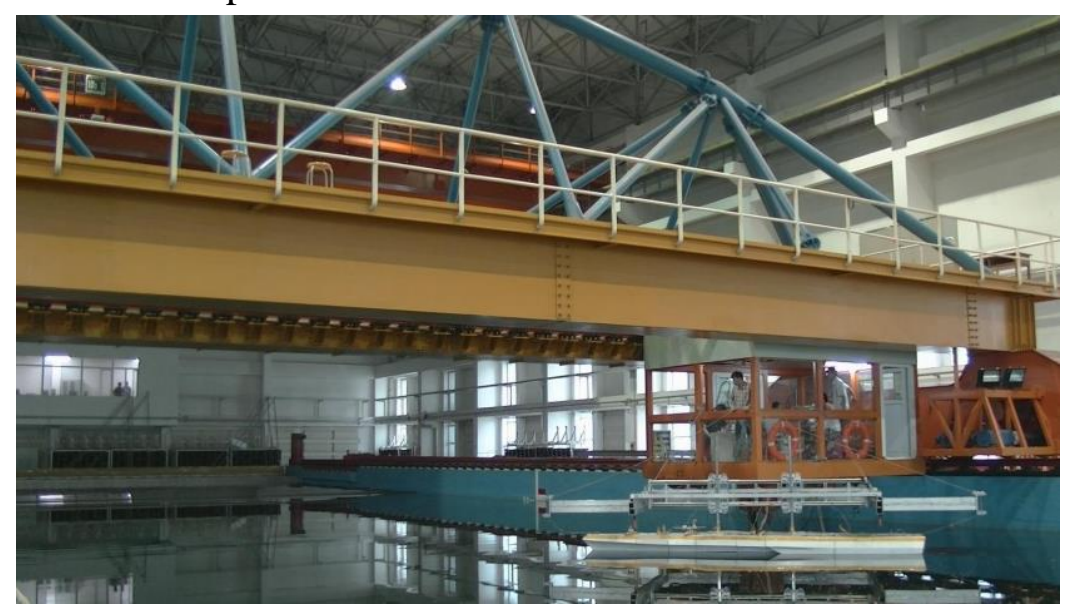

Fig. 12 Process of trimaran model test

\subsection{Model test results analysis}

\subsubsection{Preliminary analysis of structural responses}

When the trimaran model suffers wave impact in the irregular waves, the real-time structural deformation at different sections is recorded by the monitoring system. The realtime monitoring data is converted by the similitude rule and then is taken into the statistical analyses in trimaran monitoring system.

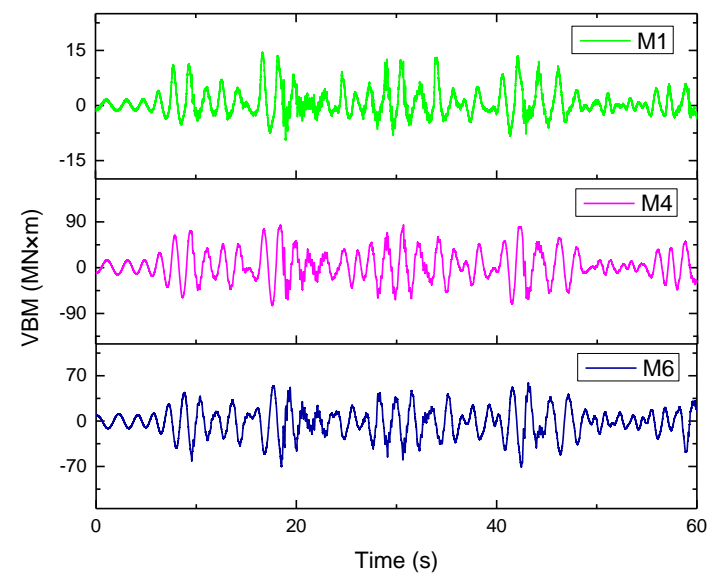

(a) VBM along trimaran

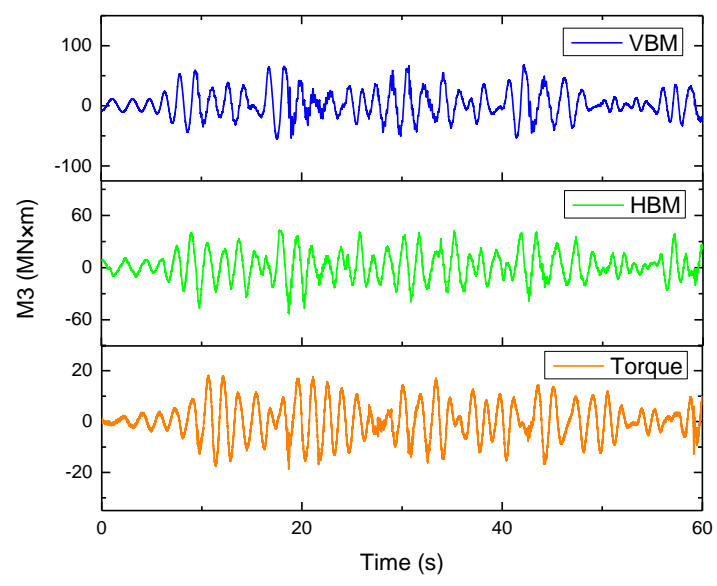

(b) Structural response at M3

Fig. 13 Time histories of structural response in bow sea 
Figure 13 (a) shows the vertical bending moment along the ship length in bow sea condition. Obviously, the real-time VBM fluctuations at these three positions are similar. And the time of max peak appearing in these positions is not far. Also, it is not hard to find that the VBM data at M1 and M6 contains more high-frequency fluctuation, although the mean amplitude of VBM at M4 is biggest in these monitoring positions. In fact, the wave slamming at bow and stern often causes the structural high-frequency vibration. And the self-propeller equipment exacerbates the high-frequency vibration at stern. In Figure 13 (b), the three kinds of structure deformations at M3 are also shown. It is found that the structural response of horizontal bending deformation and torsional deformation fluctuate more frequently than that of the vertical bending deformation in oblique waves. In addition, the structural response amplitude of VBM amidships is still bigger than amplitude of horizontal bending moment (HBM) and torque in bow sea case.

In fact, the measured structural responses are often random and irregular, when trimaran is sailing in irregular waves. Therefore, the statistical characteristic value is always used as an indicator to judge the severity of structural response in the whole irregular wave case. The autocorrelation function based on spectral analyses was conducted to obtain the significant amplitude values in monitoring system. And the results of significant amplitude values with different wave azimuths and deformation forms are shown in Figure 14. The wave azimuth of heading wave condition is defined as $180 \mathrm{deg}$. And the wave azimuth in following wave is 0 deg. Similarly, the wave azimuth case at $135 \mathrm{deg}$ is representative of the bow sea condition. And the wave azimuth of quartering sea case is $45 \mathrm{deg}$.

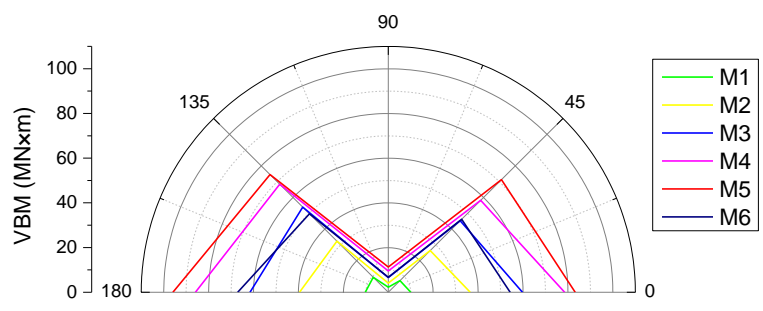

(a) VBM in different wave azimuths

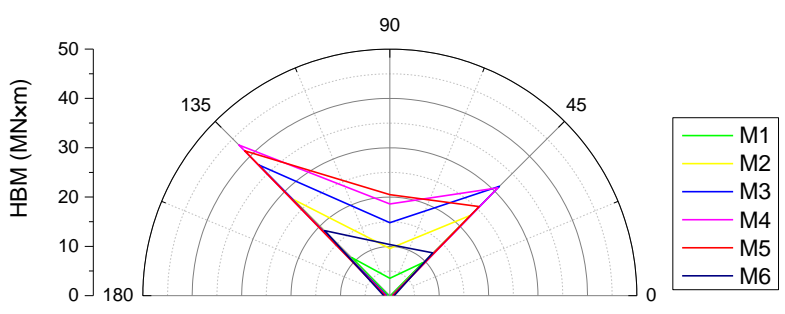

(b) HBM in different wave azimuths

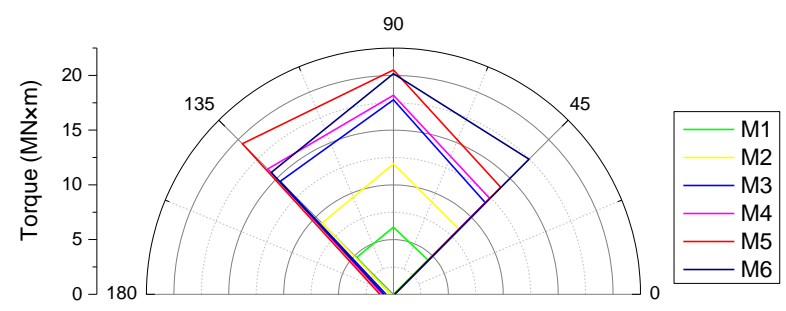

(c) Torque in different wave azimuths

Fig. 14 Structural responses with different wave azimuths

Obviously, the VBM reaches minimum in beam sea condition, as seen in Figure 14(a). And the vertical bending deformation in bow sea is more severe than the deformation in quartering sea. The severest vertical bending deformation is found at M5, it is the bow end of the cross-bridge. In fact, the flow field interference of side hull, especially wave slamming at side hulls, aggravates the vertical bending deformation at M5. And the position of max vertical bending deformation is moved a little toward stern. In Figure 14(b), it is observed easily that the trimaran in bow sea suffers more horizontal bending deformation among these irregular wave cases. The position of max HBM is not stable and changes with different wave azimuths. In bow sea condition, the HBM get the max peak at M5 like vertical bending moment. And the max HBM position is replaced by M3 in quartering sea. The arrangement of 
side hulls at stern improves the structural strength and weakens the structural response caused by wave impact from stern direction, in some extent. The statistical characteristic value of torque in different wave azimuths is also shown in Figure 14(c). Through observation, it is found that the trimaran will suffer more torsional deformation when ship is sailing in beam sea. And the position of M5 and M6, whose location contains the side hulls, get more violent torque in irregular waves. It is explained that the unique structural features of side hulls make wave force asymmetric along the width, and causes more violent torsional deformation in oblique irregular waves, especially in beam sea.

\subsubsection{Analysis of longitudinal structural strength assessment results}

The preliminary analysis mainly focuses on the structural response caused by wave impact, so it just can indicate the hull structure condition roughly. And the varied structural resistance ability is also another indispensable factor to determine structural damage risk. In fact, it is high possible to appear this phenomenon that the structure break causes by low structure resistance, although the structure suffers small wave load. In order to understand real-time hull structural condition more exactly, the differences of the longitudinal structural resistance ability are needed to be considered. The longitudinal structural strength assessment combines the wave load and structural resistance ability to evaluate the hull structural condition, and it is regarded as a more comprehensive mean to monitor the real-time hull structural condition.

The trimaran monitoring system takes the longitudinal strength thread to evaluate the structural longitudinal strength along ship length every 30 minutes. Figure 15 shows the results of this longitudinal strength assessment in 2 hours. Because the horizontal bending and torsional deformation in heading sea and following sea is too low to motivate severe structural damage risk, Figure 15 (d) only shows the assessment results of VBM in non-oblique wave cases. Although there is a high structural resistance amidships, the structural failure probability at M5 is still highest in the longitudinal strength assessment of VBM. Regardless of the different wave azimuths, the dangerous section on vertical bending deformation is invariable and consistent with the peak location of load statistical characteristic value. It is explained that the violent wave load response is still the main factor to cause the high failure probability at the most dangerous position of vertical bending deformation. But the position of second severest wave load response (M4) doesn't always get the second highest failure probability with the comparison of VBM's assessment results in different wave azimuths. In fact, the position M3 has relative lower structural resistance and is evaluated as second dangerous position in bow sea and following sea, as shown in Figure 15(a) and Figure 15(d). Therefore, the influence of low structural resistance toward the failure probability is distinct at the position with mild wave load response. In addition, the highest failure probability at M5 is happened in heading sea condition, so the heading sea condition is regarded as the most dangerous structural case for vertical bending deformation in irregular waves.

For horizontal bending deformation, the highest failure probability is found at M3 in different oblique wave cases. It is also the comprehensive result of wave load response and structural resistance. Although the position M3 is not always suffered severest wave load response, the relative lower horizontal bending structural resistance make this section more perishable at horizontal. In bow and quartering sea, the position of weak horizontal bending resistance (M2) often achieves high hull structural failure probability. And the violent wave load response at M5 also makes the structure amidships more fragile in beam sea. By observing the failure probability of horizontal bending moment in Figure 17, it is found that the highest failure probability of horizontal bending deformation is $1.86 \%$. And it is happened in bow sea. Therefore, the bow sea condition is regarded as the most concerned case for horizontal bending deformation. 


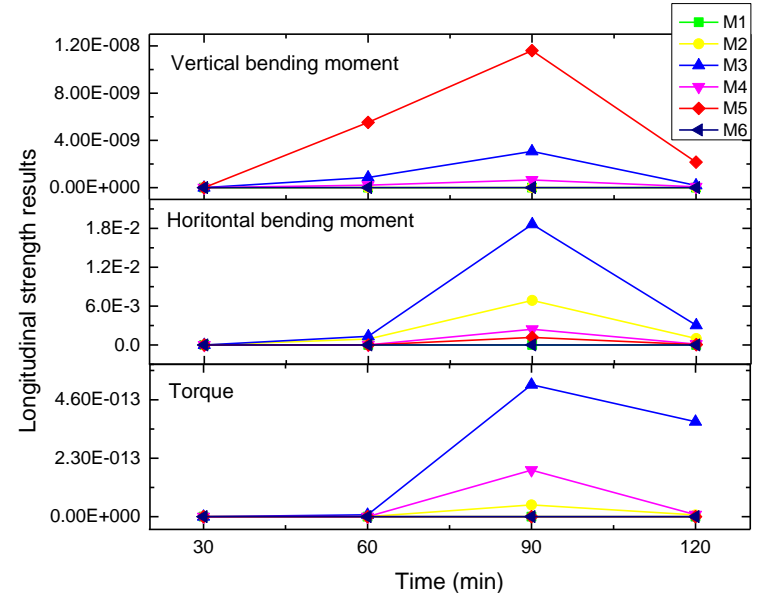

(a) Bow sea

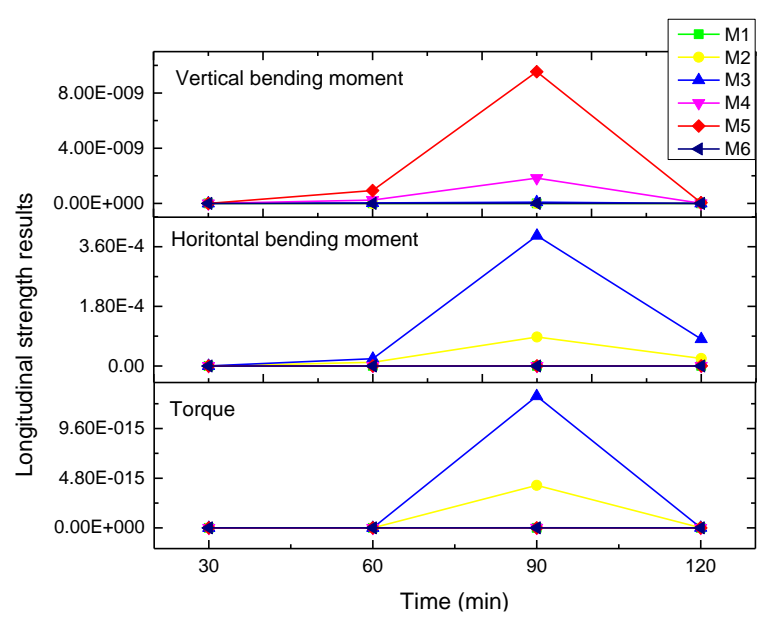

(c) Quartering sea

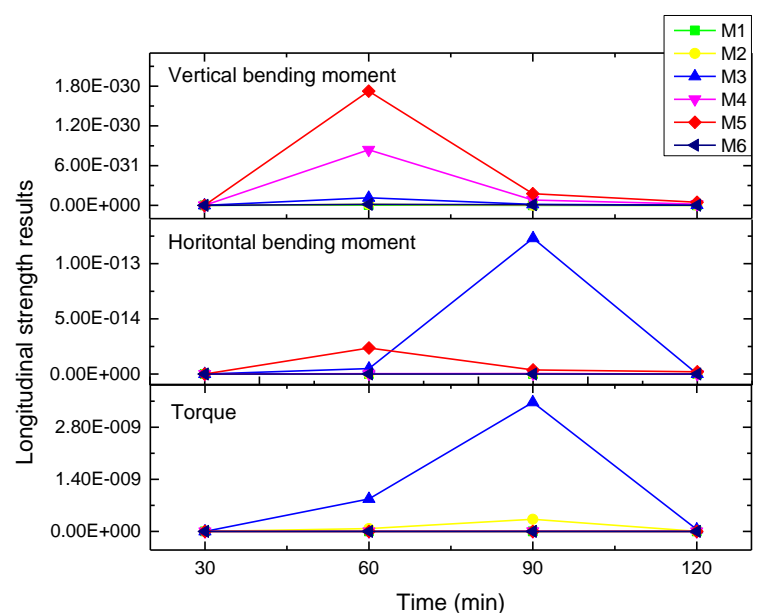

(b) Beam sea

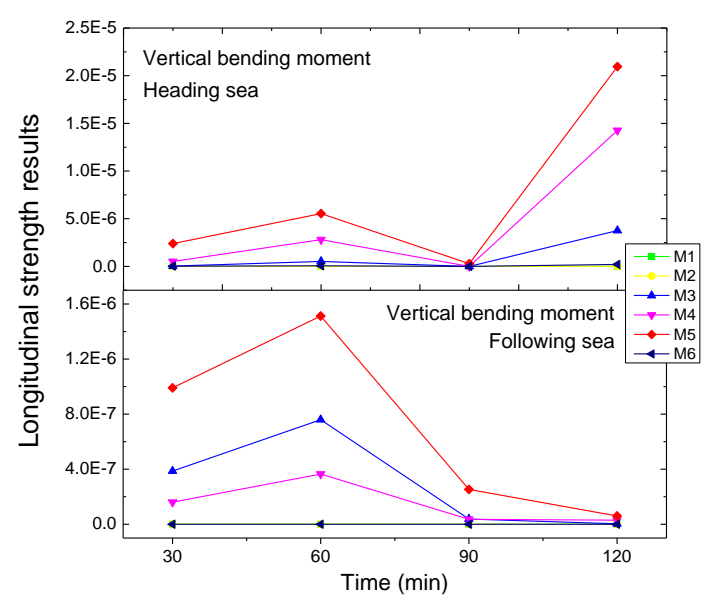

(d) Non-oblique wave

Fig. 15 Results of longitudinal strength assessment in different wave azimuths

The hull structural strength assessment of torsional deformation is also important in oblique irregular waves. Owing to the structure of side hulls and cross bridge, the trimaran gets more wave load along ship width and suffers severe torsional deformation in oblique wave cases. Although the structure at stern suffers relatively severe deformation, M3 is still the most dangerous position on torsional deformation by observing the results of structural strength assessment in monitoring system. Obviously, the common influence between wave load response and structural resistant strength makes the dangerous position move toward bow. In addition, the high failure probability at M2 also indicates the importance for structure design on torsion resistance near bow. The highest failure probability of torque is 3.47E-09, and it is found in beam sea. So, the most dangerous case for torsional deformation is beam sea. In oblique waves, the peak of failure probability on HBM and torque is often at the same time period. It suggests that the wave load response on torsional and horizontal deformation is associated lightly in time domain. By comparison of the failure probability on these three destruction forms, it is also found that the failure probability of HBM is higher than the failure probability of other deformation in oblique wave cases, although the significant amplitude value of HBM is lower than that of VBM in bow sea and quartering sea. And the failure probability of torque is relatively low among most irregular wave cases. Thus, it is 
known that the structural horizontal deformation is dangerous for the present trimaran design in oblique waves, while the torsional deformation on structure is relatively safe.

\subsubsection{Analysis of simplified yield strength assessment results}

The strain on steel girders is also monitored by the structural monitoring system for the simplified yield strength assessment and fatigue strength assessment. And Figure 16 shows the time histories of strain amidships in quartering sea.

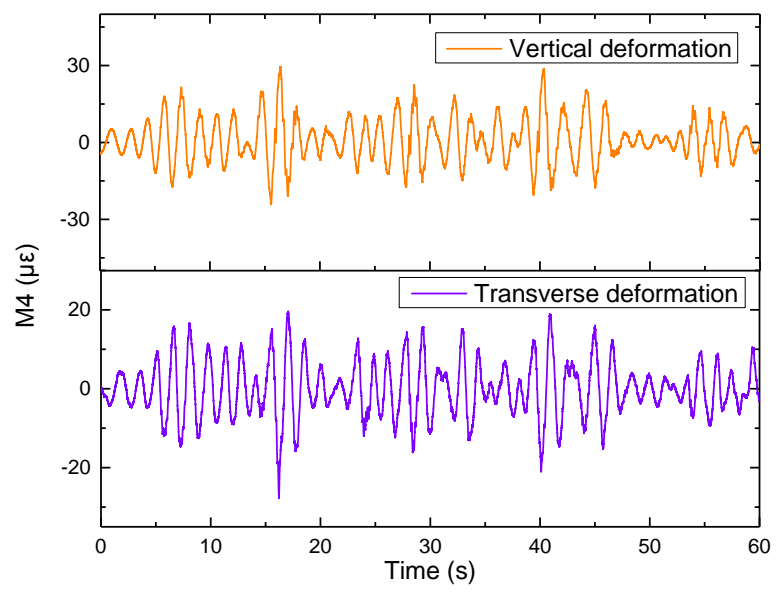

Fig. 16 Time histories of strain in quartering sea

Obviously, the similar irregular oscillation on strain is recorded in vertical deformation and transverse deformation. At the same monitoring position, the peak appearing time of strain on these two deformations is close. The real-time strain is converted to stress in monitoring system. And the simplified yield strength assessment is taken to evaluate the instantaneous structural state. Figure 17 shows final results of simplified yield strength assessment in different wave azimuths.

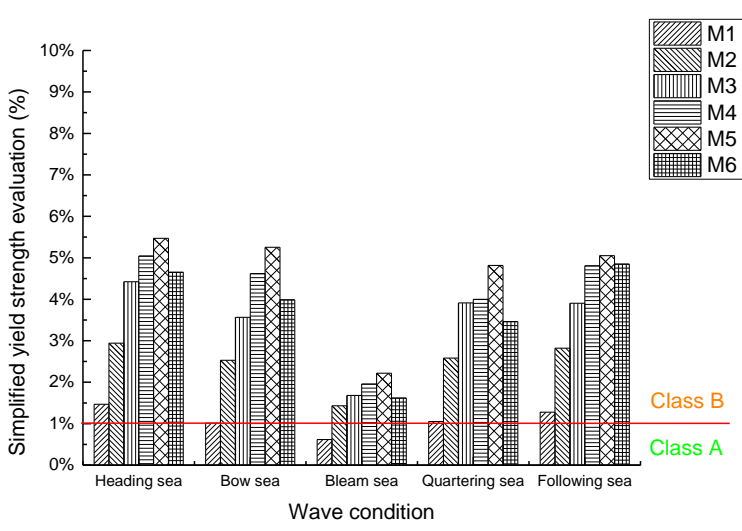

(a) Vertical deformation

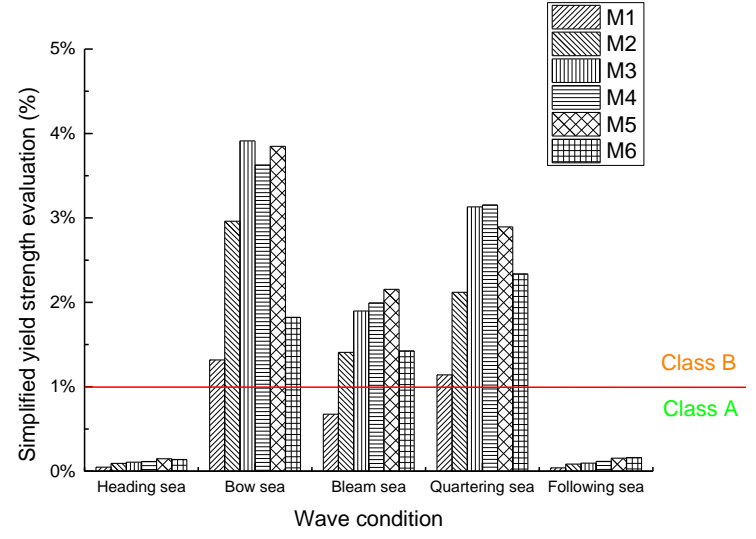

(b) Transverse deformation

Fig. 17 Simplified yield strength assessment results

In fact, the simplified yield strength assessment results both on vertical and transverse deformation fall into the region of Class A and Class B. In Figure 17(a), it is not hard to found that the vertical instantaneous yield indictor at M5 is always highest in different wave azimuths. And the max value of simplified yield strength assessment result is $6.51 \%$. The bow is least possible to have yield deformation in irregular waves, especially in beam sea. The 
instantaneous yield indictor at M1 falls into Class A, only in beam sea case. For structural transverse states, the instantaneous yield indictor of all positions in heading sea and following sea is in Class A, as seen in Figure 17(b). Non-oblique irregular wave case is too hard to motivate yield destruction on transverse. The max value of instantaneous yield indictor on transverse is $3.28 \%$ at $\mathrm{M} 3$, and it is caught in bow sea. But this transverse dangerous position is varied and changed as M5 in beam sea. By comparison in assessment results of vertical and transverse deformation, the transverse instantaneous yield indictor is still smaller than the vertical indictor. The instantaneous structural break should be paid more attention on vertical deformation.

\subsubsection{Analysis of fatigue strength assessment results}

Fatigue failure is a kind of imperceptible structural failure form. By the potential damages accumulation in long term, the flaw initiates and propagates on the surface of critical structure. With the development of flaw, the hull structure will lose its own structural resistance and break down by small wave impact in final. Therefore, in order to find the structural fatigue failure early, the critical structure of trimaran needs to be monitored in long term. And potential damages should be predicted and warned toward crew. Based on realtime monitored strain, the fatigue failure damage is calculated by the fatigue strength assessment thread in monitoring system every 5 minutes. The process of fatigue damage accumulation at vertical and transverse surface of longitudinal steel backbone is recorded in 2 hours. And these results of fatigue strength assessment on vertical and transverse are shown in Figure 18 and Figure 19, respectively.

In Figure 18, it is found easily that all time histories of fatigue accumulative damage in different wave azimuths are not linear relationship. It is obvious that the randomness of wave impact in irregular waves brings the inhomogeneous increasing on fatigue accumulative damage. Although the increased amplitude of fatigue accumulative damage in different monitoring positions is diverse, the increasing trend on fatigue accumulative damage is consistent roughly in time domain. In quartering sea, the fatigue accumulative damage at all positions increases quickly at the time period from $60 \mathrm{~min}$ to $65 \mathrm{~min}$, as seen in Fig.18 (d). The similar phenomenon can be also found in other irregular waves cases. By comparison of vertical fatigue accumulative damage in different monitoring positions, it is found that the vertical fatigue accumulative damage at M4 increases most fast in beam sea, and this damage fastest increasing position is replaced by M5 in other azimuth cases. In fact, the position M5 always obtain higher amplitude of strain on vertical deformation than other positions in irregular waves. Therefore, the vertical fatigue accumulative damage at M5 enhances acutely in most irregular wave cases. The position M4 suffers more cycle number of vertical strain than the cycle number at M5 in beam sea, and strain amplitude gap between M4 and M5 is not far. Thus, the M4 finally become the dangerous position of fatigue strength in beam sea. This difference of strain cycle number is also explained by the phenomenon that the trimaran cross-bridge structure bring some structural vibration into the main hull, when the side hull blocks the wave spreading in beam sea.

Figure 18 (e) shows the final fatigue accumulative damage at four dangerous positions. Obviously, the max value of fatigue accumulative damage is happened at M5 in heading sea. So the position at the start end of side hulls in heading sea should be considered carefully in the design of structural fatigue resistance. Although the amplitude of strain on vertical deformation at M3 is higher than the strain amplitude at M2, the bow slamming motivate more higher frequency vertical vibration and make M2 suffers fatigue damage more violently than M3 by comparing the fatigue damage in Figure18(a)-(d). Thus, the influence of high 
frequency vibration cause by wave slamming is also important in fatigue strength assessment and cannot be ignored.

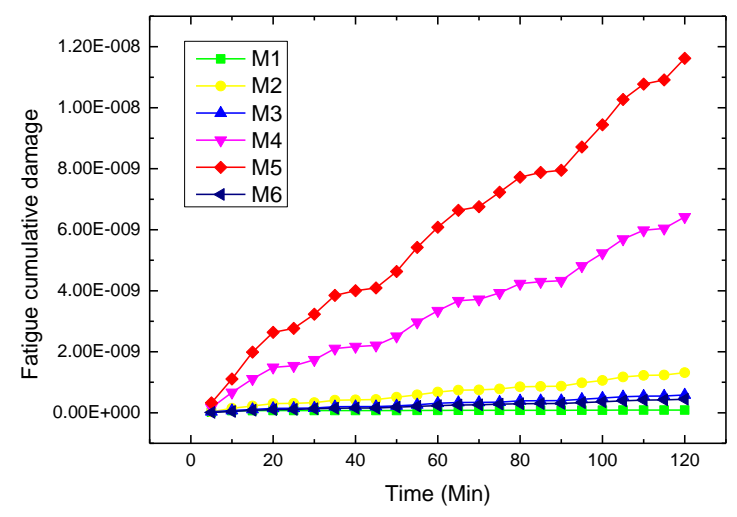

(a) Fatigue damage in heading sea

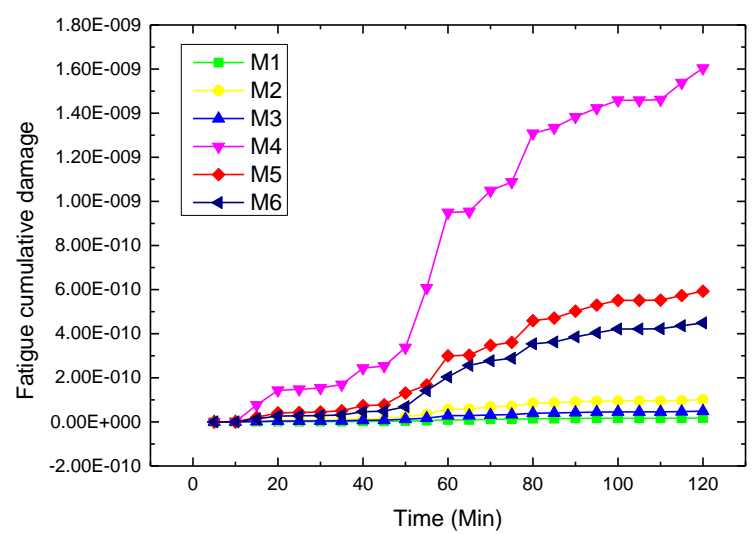

(c) Fatigue damagein beam sea

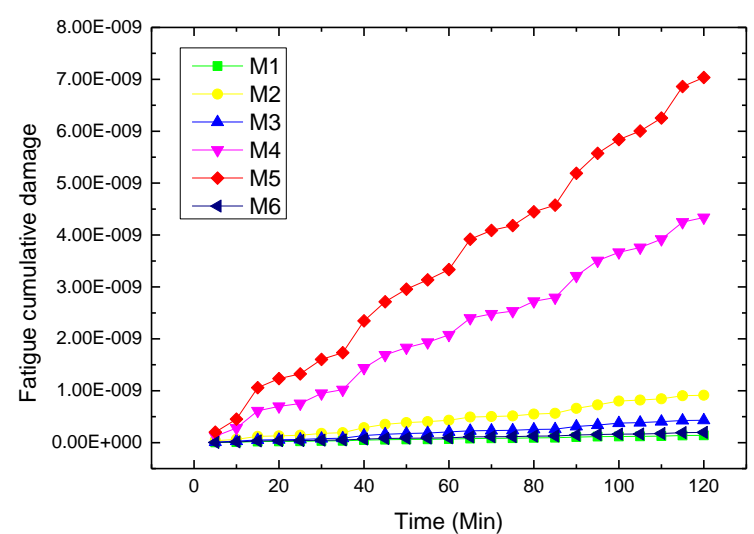

(d) Fatigue damage in following sea

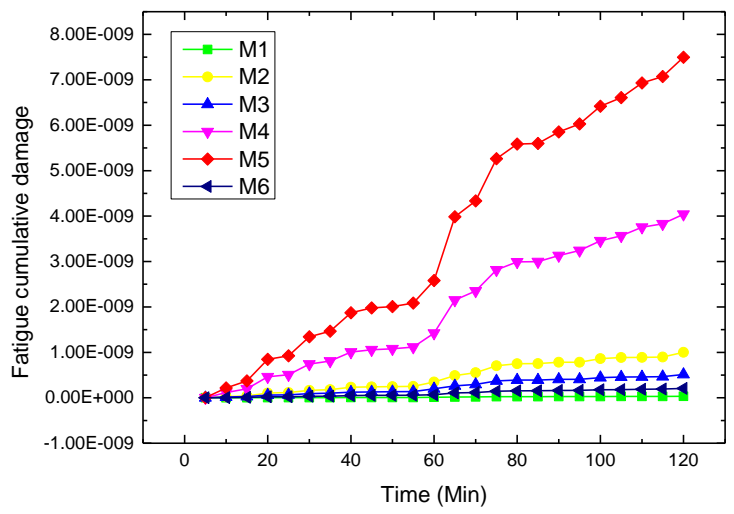

(b) Fatigue damage in bow sea

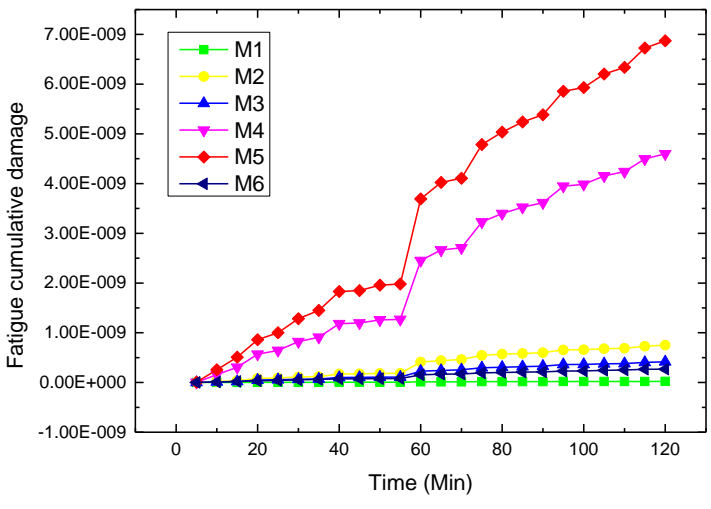

(d) Fatigue damagein quartering sea

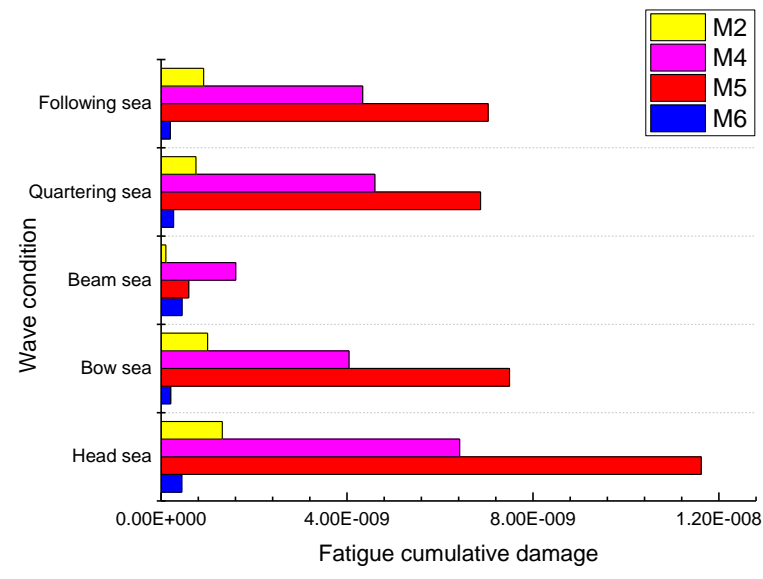

(e) Final fatigue damage results

Fig. 18 Vertical fatigue strength assessment

Because of weak transverse vibration in head sea and following sea, the structural fatigue breaks on transverse is too hard to form. Therefore, Figure 19(d) only shows the results of transverse fatigue strength assessment in oblique waves. 


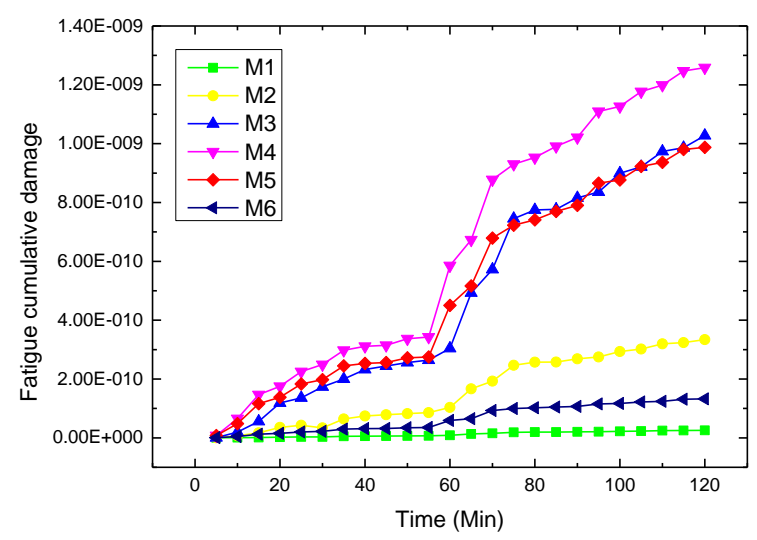

(a) Fatigue damage in bow sea

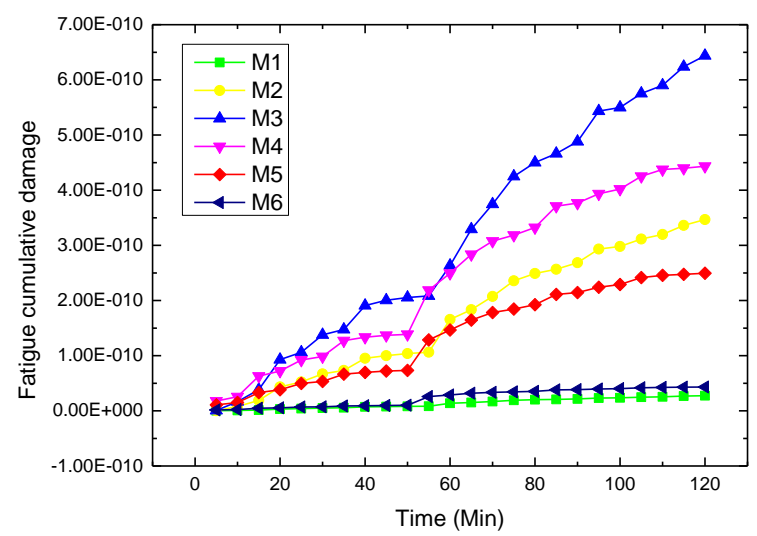

(c) Fatigue damage in quartering sea

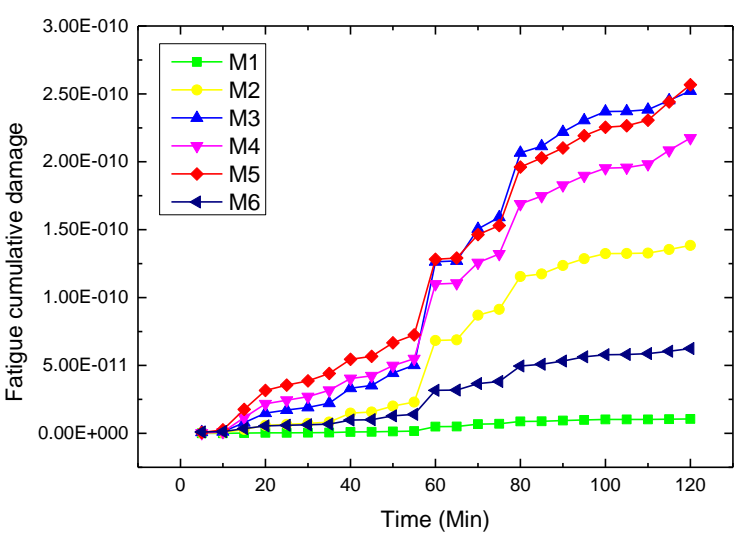

(b) Fatigue damage in beam sea

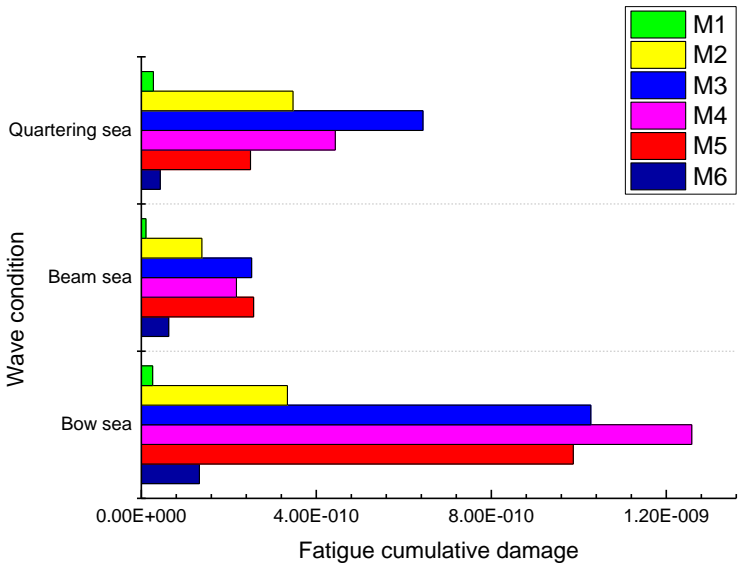

(d) Final fatigue damage results

Fig. 19 Transverse fatigue strength assessment

Through observation of the transverse fatigue cumulative damage at different monitoring locations, it is found that the gap of transverse fatigue damage among M3, M4 and M5, is not far. And the highest fatigue damage appears at these three positions alternately in quartering sea, bow sea and beam sea. Obviously, the most dangerous position on transverse fatigue breaks is unfixed, and the high fatigue damage position is sensitive with wave azimuths. Even in same wave condition, the high fatigue damage position has high possible to change at different times. In the beam sea case, the M3 replace M5 as highest fatigue damage position at $70 \mathrm{~min}$, but the situation turn back at $120 \mathrm{~min}$. This replacement of max dangerous position is observed clearly in Fig.19 (b). In quartering sea, the similar fluctuation is also found by comparing the fatigue damage of M3 and M4 in Figure19 (c). The bow sea condition is considered as the most dangerous cases for transverse fatigue damage by the final fatigue damage statistic results in Figure 19 (d). And the transverse position at M4 should be focused as the important location to avoid the transverse structural fatigue breaks.

\section{Conclusions}

In this paper, a kind of structural monitoring and assessment system for trimaran is designed and installed in a self-propelled model to study the fluctuation characteristics of trimaran structural state in irregular waves. The trimaran model is equipped with some strain 
sensors along the ship length and tested in different wave azimuths. Meanwhile, three realtime structural strength assessment methods are adopted in the monitoring system to evaluate the trimaran structural state at different monitoring locations and irregular wave cases. The final results of these structural strength assessments in the trimaran monitoring system is compared and analysed. And the conclusions are made as follows:

(1) The structural monitoring and assessment system for trimaran is proved to achieve well the purpose of trimaran structure safety monitoring by a series of model tests. The realtime strain and sectional indicator is monitored and stored in the system database successfully. And the simplified yield strength assessment in the system can record instantaneous structural state effectively. The overall and local structural condition of trimaran is also evaluated autonomously in the system module of longitudinal and fatigue strength assessments. And all these monitoring and assessment data by this monitoring system will give the foundation of the actual trimaran structural monitoring.

(2) The location at bow end of side hull is regarded as the most dangerous position for vertical bending deformation. Although the distribution of longitudinal structural resistance is considered in longitudinal structural strength assessment, the violent wave load response is still found as the main factor to determine this dangerous position of vertical bending deformation. But, for horizontal bending and torsional deformation, the structural resistance has played an unignored role on selecting the dangerous position. In longitudinal structural strength assessment of horizontal bending moment and torque, the trimaran arrangement of side hull at stern improves the stern structural resistance and decreases structural failure possibility at stern. Therefore, the dangerous position of horizontal bending and torsional deformation is near to the trimaran bow relatively.

(3) In simplified yield strength assessment of the monitoring system, the instantaneous yield indictors in both vertical and transverse deformation fall into Class A and B. It is suggest that this trimaran structural instantaneous condition is relatively safe. The peak of vertical yield indictor is always observed at the bow end of side hull and unvaried at different environments, while the peak location of transverse yield indictor is changed with different wave azimuths. Obviously, the structural yielding failure on the local structure of trimaran is sensitive to wave azimuths. Thus, the probability distribution of wave direction encountered by trimaran should be considered in the specific selection of structural yield strength monitoring points for the full-scale trimaran structural monitoring.

(4) In irregular waves, the increasing of fatigue accumulative damage in fatigue strength assessment are not linear, but the increasing trend of fatigue damage at different monitoring positions is consistent roughly on time domain. The fastest vertical fatigue accumulative damage is also found at bow end of side hull. And the dangerous position of horizontal fatigue damage in oblique waves is varied in the midship region. For the horizontal structural fatigue failure, the bow sea case should be taken more attention during the trimaran voyage.

In present research, the main monitoring subjects focus on the trimaran longitudinal positions. The cross-bridge structural monitoring and wave impact will be considered in further research. And the inverse finite element method will be adapted to predict of the local structural stress [23-24]. The full-scale or large-scale trimaran test in actual sea environment will be also taken to make the monitoring technology more credible.

\section{ACKNOWLEDGMENTS}

Special thanks to all members in ship structural mechanics laboratory of Harbin Engineering University and Dalian Maritime University for the preparation of this trimaran test. This research was supported by the National Natural Science Foundations of China 
(No.51679049 and No.51709030) and the Fundamental Research Funds for the Central Universities (3132019134). The authors express their gratitude to these foundations.

\section{REFERENCES}

[1] K. Lindemann, J. Odland, J. Strengtheagen: On the application of hull surveillance systems for increased safety and improved structural utilization in rough weather. Transactions of SNAME, Vol.85, pp131-166, (1977).

[2] G.X. Li: Joint development of hull monitoring system in Europe. Ships \& Yachts, Vol.11, pp40, (1994).

[3] D.R. Hjelme, L. Bjerka, S. Neegard, et al: Application of Bragg grating sensors in the characterization of scaled marine vehicle models. Applied Optic, Vol.36, pp328-336, (1997). https://doi.org/10.1364/AO.36.000328

[4] G Wang, K Pran, G Sagvolden, et al: Ship hull structure monitoring using fiber optic sensors. Smart Materials and Structures, Vol.10, pp472-478, (2001). https://doi.org/10.1088/0964-1726/10/3/308

[5] P. Karianne, B.H Geir, G. Sagvolden, et al: Wavelength multiplexed fiber Bragg grating system for highstrain health monitoring applications. Measurement Science and Technology, Vol.13, pp471-476, (2002).

[6] G. Sagvolden, K. Pran, L. Vines, et al: Fiber optic system for ship hull monitoring. The 15th optical fiber sensors conference technical digest, Vol.1, pp435-438, (2002).

[7] S.P. Huston: Structural health monitoring of a high speed naval vessel using ambient vibrations. Georgia Institute of Technology, 2010.

[8] N.M. Okasha, D.M. Frangopol, D Saydam, et al. Reliability analysis and damage detection in high-speed naval craft based on structural health monitoring data. Structural Health Monitoring, Vol.10, pp361-379, (2011). https://doi.org/10.1177/1475921710379516

[9] U.D. Nielsen, J.J. Jensen, P.T. Pedersen, et al: Onboard monitoring of fatigue damage rates in the hull girder. Marine Structures, Vol.24, pp182-206, (2011). https://doi.org/10.1016/j.marstruc.2011.03.003

[10] I. Renato, J. Irena, M. Zoran: Longitudinal ship's hull strength monitoring with optical fiber sensors. ELMAR 51st International Symposium, pp167-170, (2009).

[11] J.E. Kokarakis, J. Goyet, G.D. Jong, J.F. Segretain: Predictor-corrector system for health monitoring of the hull structure of bulk carriers. Royal Institution of Naval Architects-International Conference on Design and Operation of Bulk Carriers, pp145-159, (2009).

[12] W.B Xu, C.L. Yang, X.Y. Shi: Design and implementation of vessel structure safety monitoring system based on CAN bus. Journal of Dalian Maritime University, Vol.32, pp17-20, (2006).

[13] J. He: Real time monitoring system for ship deck deformation. Ship science and technology, Vol.29, pp45-48, (2007)

[14] Y.X. Jin, X. Hu, C.J. Shi: Supervision and evaluation system of container vessel hull structure. Journal of Shanghai Maritime University, Vol.29, pp1-4, (2008).

[15] G.G San: Application of structural reliability theory ship longitudinal strength. Shipbuilding. Vol.4, pp102-123, (1986).

[16] Lloyd's Register of Shipping: Rules for the Classification of Trimarans, Lloyd's Register Rules and Regulations, 2006.

[17] C.F. Li: Residual Strength Assessment of Damaged Warships, Doctoral thesis, Harbin Engineering University, 2009.

[18] J.H. Liu: The Research on Low Cycle Fatigue Fracture Properties of 304 stainless steel under Tensile and Compressive loading, Master thesis, Lanzhou University of Technology, 2011.

[19] China Classification Society: Guidelines for Fatigue Strength of Ship Structure, China Classification Society Press, 2015.

[20] W.P. Zhang, Z. Zhong, S.L. Ni: Model testing of seakeeping performance of trinaran. Journal of hydrodynamics A, vol.22, pp.619-624, (2007).

[21] Z.Y. Chen, H.L. Ren: Wave load experimental investigation of a segmented model of a very large ship based on variable cross-section beams. Journal of Harbin Engineering University, vol.33, pp263-268, (2012).

[22] J.L. Jiao, S.Z. Shun, H.L. Ren: Predictions of wave induced ship motions and loads by large-scale model measurement at sea and numerical analysis. Brodogradnja/Shipbuilding, vol.67, pp81-100, (2016). https://doi.org/10.21278/brod67206 
[23] A. Kefal and E.Oterkus: Displacement and stress monitoring of a Panamax containership using inverse finite element method. Ocean Engineering, vol.119, pp16-29, (2016). https://doi.org/10.1016/j.oceaneng.2016.04.025

[24] A. Kefal and E.Oterkus: Displacement and stress monitoring of a chemical tanker based on inverse finite element method. Ocean Engineering, vol.112, pp33-46, (2016).

https://doi.org/10.1016/j.oceaneng.2015.11.032

Submitted: $\quad$ 27.03.2017. Haoyun Tang ${ }^{1}$, conanthy@126.com

Huilong Ren

Accepted: 26.04.2019. Qi Zhong ${ }^{2}$

${ }^{1}$ Navigation College, Dalian Maritime University, No.1 Linghai Road, Hgih tech Zone, Dalian City, Liaoning Province, China

2 College of Shipbuilding Engineering, Harbin Engineering University,

No.145, Nantong Street, Harbin, China 